\title{
Application of Remote Sensing Technology in Engineering Geological Surveying and Surveying
}

\author{
Kai Guo \\ The Second Geological Team of Hebei Bureau of Geology and Mineral Resources, Tangshan, Hebei, 063000, China
}

\begin{abstract}
In engineering geological surveying and mapping, in order to better meet the requirements of engineering design and construction, engineering geological surveying and mapping generally uses specialized surveying and mapping techniques. With the gradual and widespread application of modern surveying and mapping technologies, the technical methods and means of geological surveying and mapping will gradually be replaced. In particular, geological surveying and mapping work is an important part of geological surveying and mapping work, which can effectively improve the efficiency and accuracy of geological surveying and mapping work, thereby avoiding errors caused by human records, especially when conducting research and analyzing the distribution of rock formations and minerals in the area. Only by using image positioning technology can we accurately classify the minerals and rock formations in the area, determine the basic attributes of their geology, ensure the progress of geological surveying and mapping, and reduce the consumption of human resources. Therefore, the paper analyzes the application of remote sensing images in geological surveying and mapping, in order to promote the improvement of the technical level of geological surveying and mapping. Keywords: Remote sensing technology; Engineering geological surveying and mapping; Surveying and mapping technology
\end{abstract}

Citation: Kai Guo, 2021. Application of Remote Sensing Technology in Engineering Geological Surveying and Surveying. International Journal of Geology, 6(1): 21-24. http://doi.org/10.26789/IJG.2021.01.005

Copyright: Application of Remote Sensing Technology in Engineering Geological Surveying and Surveying. (C) 2021 Kai Guo. This is an Open Access article published by Urban Development Scientific Publishing Company. It is distributed under the terms of the Creative Commons Attribution-Noncommercial 4.0 International License, permitting all non-commercial use, distribution, and reproduction in any medium, provided the original work is properly cited and acknowledged.

\section{The Meaning of Remote Sensing Technology}

Remote sensing and telemetry are the abbreviations for remote sensing and remote measurement. Remote sensing technology is to measure and record the nature and characteristics of distant objects through highly sensitive instruments and equipment. The basic theory it is based on is the electromagnetic wave theory. Specifically, it obtains the necessary geological and geomorphological information by observing the electromagnetic spectrum emitted (or reflected) by the terrain and ground objects near the surface, so as to provide a basis for solving related problems. The basic principles of electromagnetic wave detection of geological bodies are:

(1) Any geological body has the ability to selectively absorb and reflect electromagnetic waves. For example, a geological body absorbs electromagnetic waves from sunlight while emitting electromagnetic waves during the day. At night, in addition to the electromagnetic waves emitted by the geological body itself, it also radiates the electromagnetic waves absorbed during the day. Generally speaking, if a geological body has the transmitting ability of a certain band, it will also have the absorbing ability of that band. The remote sensing technology is to use the difference of electromagnetic waves emitted, reflected and absorbed by different geological bodies to identify geological bodies. At present, people have done certain research on the characteristics of the emission spectrum, reflection spectrum, and absorption spectrum of geological bodies, and they have been successfully used in the interpretation of remote sensing data.

(2) Any geological body has the ability to emit electromagnetic waves. Physics research shows. Any object can emit electromagnetic waves when the temperature is greater than $-273.16^{\circ} \mathrm{C}$. And different geological bodies, due to the difference of their material structure. The wavelength ranges of the electromagnetic waves emitted by them are different. On the other hand, the intensity and wavelength of electromagnetic waves emitted by an object are related to the temperature of the object itself. For example, the same object emits infrared light when 
the temperature is low, and can emit visible light when the temperature rises to a certain level.

Remote sensing and telemetry instruments are generally installed on aircraft for observation. Since the earth is observed from a long distance away from the earth, phenomena that cannot be observed or cannot be seen clearly on the earth can be observed more objectively and comprehensively. Because it can observe a wide area at the same time, it is possible to grasp the geological and geomorphological conditions of the survey area from a macro perspective, and because the data of a large area can be quickly obtained at the same time, it is beneficial to achieve multiple repeated measurements. The comparison of data obtained many times can reflect the dynamic changes of geological phenomena. This is very important for engineering geological survey research. For example, it is very suitable for monitoring large-scale landslides, the dynamics of mud-rock flows, the effects of rivers, the changes of shorelines, and the identification of regional structural skeleton characteristics

\section{Application of Remote Sensing Technology in Engineering Geological Surveying and Surveying}

There are two recording methods for remote sensing data. One is non-imaging, which means recording numerical and curve data on tape; the other is imaging, which converts surveying and mapping data into images through photographic imaging, scanning imaging, and holographic imaging. At present, there are many applications of imaging methods. Among them, aerial photography and satellite photos are the most important remote sensing technical data to interpret aerial photos, mainly to analyze their morphological characteristics, tones, shapes, sizes, shadows and distribution. Due to the limitation of the aircraft's flying height and the center projection, the edge part of the aerial photos will be greatly distorted, and attention should be paid when interpreting. Satellite photos are taken at high altitude and the image area is small, so they can be approximated as vertical projections, which can overcome the defect of edge distortion of aerial photos. However, satellite photos generally have a small scale and low resolution.

\section{Application of Aerial Photos in Engineering Geological Surveying and Mapping}

\subsection{The Scope of Application of Aerial Photos}

Aerial photos are suitable for the selection of railways and highways, and the remediation of geological disasters. It is adopted in the stages of river basin planning and water conservancy project site selection. Especially for areas where geological information is lacking, such as difficult-to-traffic mountainous areas and inaccessible border areas, the use of aerial photos to interpret the engineering geological conditions has unique advantages. Even in areas with sufficient geological data, the use of aerial photos is conducive to understanding the geological conditions related to engineering construction on a large scale, making the obtained geological data of the survey area more macroscopic and comprehensive, and conducive to more targeted Prospecting work. Therefore, when carrying out engineering geological surveying and mapping in areas with aerial photos, we should make full use of aerial photos.

\subsection{Engineering Geological Interpretation of Ae- rial Photos}

\subsubsection{The Engineering Geological Interpretation Steps of Aerial Photos}

The engineering geological interpretation of aerial photos is generally carried out in four steps: (1) Recognition of typical locations in the on-site work area. That is, the understanding of the imaging response of the geological conditions and characteristics of the area obtained by the photo; (2) The interpretation of the indoor photo is based on the collected data and the knowledge of the typical location in the field, plus the practical experience of the staff. Geological observation. Use theoretical knowledge for logical reasoning. Explain them; then use certain legend symbols to indicate the determined geological structure lines, stratigraphic boundaries, the development range of physical geological phenomena, and groundwater outcrops on the photo, or draw them separately on the base map; (3) Outside Industry verification verification, for the problems that cannot be solved correctly through indoor interpretation for a while, it is necessary to conduct field verification work. Through field performance verification, the original indoor interpretation undiscovered and undetermined problems were supplemented. Correct the original interpretation errors; (4) Re-judgment and data sorting indoors.

\subsubsection{Engineering Geological Interpretation Method of Aerial Photos}

The main methods of engineering geological interpretation of aerial photos are the contrast method and the neighbor ratio method. The so-called comparison method is based on the existing samples and reference materials in the work area and the on-site experience of the staff. Compare the confirmed object with the object to be studied, so as to interpret its content and essence; while the 
neighboring method mainly determines its boundary according to the difference of neighboring features.

\subsubsection{The Content of Engineering Geological In- terpretation of Aerial Photos}

(1) Interpretation of Lithology

First of all, different types of rocks must be distinguished based on the boundaries of outcrops and other signs. For example, the outcrops of sedimentary rocks are generally in the shape of bands, the outcrops of magmatic rocks are generally in the shape of blocks or veins, and the unconsolidated loose sediments are the Quaternary. Then, according to the different color characteristics of rocks of different natures, the specific nature types are determined.

(2) Explanation of structure

It is most conducive to the interpretation of the structure when the topographical cutting is relatively strong, the small and medium-sized landforms are developed, and the outcrops are good. The interpretation of rock formations is based on a certain relationship between ground ups and downs and geological structure. The horizontal rock formations often form flat-topped mountains, the outcrops of the upright rock formations are straight, and the outcrops of the inclined rock formations are often curved. If you want to determine the fold structure, you must pay attention to the mutual position of triangles, terraces and other structural elements. If it is a line and a closed loop, it may be-folds.

When the vertices of the triangles are the same, it is an anticline, and vice versa, it is a syncline. Generally, the outcropping line of high-angle faults on the surface is approximately straight, and the terrain is prone to form steep cliffs and faulted valleys. For low-angle faults, the outcrop lines are often curved, and the ground surface is mostly gentle slopes.

(3) Interpretation of topography

It can be directly judged from the topography, such as the slopes, terraces, floodplains, oxbow lakes, and karst landforms of river valley landforms. It is directly reflected in the photo.

(4) Interpretation of physical geological phenomena

It is closely related to the interpretation of geomorphology, such as alluvial fans, alluvial fans, landslides, etc. can also be directly interpreted based on geomorphology.

The accuracy of aerial photo interpretation depends on the geological and landform characteristics of the work area, the experience of the staff, and the amount of information available in the area. Practice has proved that geomorphological research through aerial photo interpretation is very effective. In addition, the use of aerial photos to observe and understand the full picture of the development of geological structures in a large area also has unique advantages.

\section{Application of Satellite Photos in Engineering Geological Surveying and Mapping}

\subsection{Features of Satellite Photos}

First of all, because the altitude of a general satellite orbit is about $905.5 \sim 918 \mathrm{~km}$, it is much higher than the flying altitude of an airplane. Therefore, the scope of satellite photos is very large, which provides favorable conditions for people to study various geological phenomena on the surface macroscopically. Avoid the limitations of ground work. Secondly, satellite photos contain a lot of image information. Generally, satellites are equipped with more than two kinds of multi-spectral remote sensors. Can obtain many spectral information photos of different bands at the same time. In this way, images of ground scenes on different bands can be obtained, so that topography and ground objects can be distinguished from the difference in image structure and the difference in spectral characteristics of different bands. This will play a key role in improving the reliability of the analysis results; in addition, satellite photos can quickly reflect various dynamic changes and can be used to study active physical and geological phenomena.

\subsection{Engineering Geological Interpretation of Sat- ellite Photos}

The interpretation of satellite photos is mainly based on two basic geological information, one is morphological feature information, and the other is tonal feature information. The morphological feature information is the phenomenon formed on the photo by the geological body reflecting the visible light in the sunlight. Even under the same lithological conditions. Due to the difference in resistance to weathering and erosion of each part of the rock mass. The surface also shows the difference of different slope and aspect. In this way, the angle of incidence and reflection are different. The morphological feature information is formed. According to the different morphological characteristics, the geological body can be identified. Tonal feature information is the result of the comprehensive effect of the reflection, absorption, and transmission of natural light sources (mainly visible electromagnetic waves emitted by the sun) on satellite photos by geological bodies.

According to the theory of electromagnetic wave geology, the tones of geological bodies are divided into two categories: colored geological bodies and achromatic geological bodies. Colored geological bodies are geolog- 
ical bodies that have the ability to selectively absorb or reflect external visible light. For example, red sandstone can absorb all the monochromatic light except red in the visible light. Only red light is reflected, so it appears red. Achromatic geological bodies do not have the ability to decompose external visible light into monochromatic light. They can only absorb or reflect all external visible light. When the geological body cannot decompose the external visible light and absorbs little and reflects a lot, the geological body appears white. Such as dolomite, gypsum, etc. On the contrary, if they absorb a lot and reflect very little, they appear black. Such as carbonaceous shale and basic igneous rock. When the geological body is in the transition state of the above two, it will show different degrees of gray (such as light gray, gray, dark gray, etc.). In the achromatic satellite photos, the tonal characteristics of all geological bodies have become the characteristics of achromatic geological bodies. The color of the geological body is represented by gray scale. The gray scale can generally be divided into 1 to 5 levels (and there are also 10 levels), and all the geological bodies are black or dark in nature. The image on the satellite photo is black or dark gray. And all geological hue whose true color is light color. The image is also light (gray). Therefore, most of the light-colored rocks such as acidic igneous rock, dolomite, marble, quartz sandstone, and quartzite have light-colored images. On the contrary, basic or ultrabasic igneous rocks have a dark image tone, but it should be noted that the depth of the image tone is not only affected by lithology, but also interfered by many other factors. For example, when the rock has high humidity, its The image tone will deepen, these factors should be paid attention to in specific analysis.

\section{The Main Existing Problems in the Application of Remote Sensing Technology}

Remote sensing technology has not yet been widely used. In the geological surveying and mapping team, people are currently relatively unfamiliar with remote sensing technology, which makes it difficult for remote sensing technology to play its due role in geological hazard surveys; remote sensing surveys of geological hazards require multi-temporal real-time or quasi-real-time remote sensing information sources. Information sources are expensive. Limited by funds, remote sensing surveys of geological hazards are difficult to popularize, and are currently limited to geological hazard surveys in key areas and key projects; currently commonly used remote sensing information sources have a small spatial resolution, which is difficult to meet the detailed survey work of geological hazards., Which makes remote sensing technology only widely used in macroscopic surveys, and less in microscopic applications. Remote sensing technology has been widely used and good results in engineering geological survey, environmental geology and geological disaster research, but it is urgent to conduct in-depth research with new ideas to improve the level of application.

\section{Conclusions}

Remote sensing technology is a new high-tech means. The use of remote sensing technology to carry out geological disaster surveys is not only necessary, but also feasible. Remote sensing technology can run through the entire process of geological disaster investigation, monitoring, early warning, and evaluation. With the gradual improvement of the theory of remote sensing technology and the continuous improvement of the spatial resolution, time resolution and spectral resolution of remote sensing images, remote sensing technology is bound to become the macro survey of geological disasters and their disaster-generating environment, as well as the dynamic monitoring of disasters and the assessment of disaster losses. One of the indispensable methods is to provide more advanced technical support and more comprehensive database materials for geological surveying and mapping, and to provide more detailed data and information for "Digital China" to comprehensively enhance the comprehensive competitiveness of the industry.

\section{References}

[1] Chen Zhonghou, Wang Xing, Tai Du Shihan. Engineering and Environmental Geophysical Prospecting Course [M]. Beijing: Geological Publishing House, 2005.

[2] Xiong Shengqing, Nie Hongfeng, Yang Jinzhong. The application of remote sensing technology in the survey and monitoring of geological disasters[A] Proceedings of the National Seminar on Emergency Response and Disaster Prevention Technology for Sudden Geological Disasters[C].2010.

[3] Li Xinsheng. The characteristics, current situation and prospects of the surveying and mapping work of the geological department[J]. Yunnan Geology, 1985(02): 18-20.

[4] Zhu Liangpu. Remote sensing image geological interpretation tutorial [M]. Beijing: Geological Publishing House, 1981. 